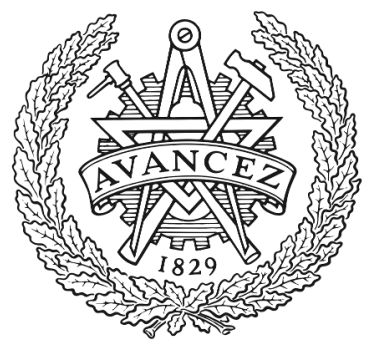

CHALMERS

UNIVERSITY OF TECHNOLOGY

\title{
Gap waveguides and PMC packaging: Octave bandwidth mm- and submm-wave applications of soft \& hard surfaces, EBGs and AMCs
}

Downloaded from: https://research.chalmers.se, 2023-04-26 12:03 UTC

Citation for the original published paper (version of record):

Kildal, P. (2013). Gap waveguides and PMC packaging: Octave bandwidth mm- and submm-wave applications of soft \& hard surfaces, EBGs and AMCs. Asia-Pacific Microwave Conference Proceedings, APMC: 34-36. http://dx.doi.org/10.1109/APMC.2013.6695182

N.B. When citing this work, cite the original published paper. 


\title{
Gap Waveguides and PMC Packaging: Octave Bandwidth mm- and submm-Wave Applications of Soft \& Hard Surfaces, EBGs and AMCs
}

\author{
Per-Simon Kildal
}

\author{
Department of Signals and Systems, Chalmers University of Technology, 41296 Gothenburg, Sweden
}

per-simon.kildal@chalmers.se

\begin{abstract}
AMCs, EBGs and other surface-based metamaterials are known to have narrow bandwidths. However, when they are used to generate stopbands for parallel-plate modes, the bandwidth can be very large. This characteristic is used in the gap waveguide technology that was invented in 2008, based on old research on soft and hard surfaces. The gap waveguide technology can be used to package microstrip and CPW circuits, but can also with advantage replace such standard technologies and in particular above $30 \mathrm{GHz}$. The gap waveguides can have similar lowloss performance as solid rectangular waveguides, but they can be realized in a much better way. Rectangular waveguides are normally realized by split blocks which are screwed tightly together to ensure good conductive contact, whereas gap waveguides can be realized between parallel plates without metal connection. This paper overviews how the gap waveguide technology has been explored during the passed five years including: demonstrations of the wideband lowloss guiding characteristics up to $260 \mathrm{GHz}$, demonstrations of packaging in different frequency ranges with different AMCs or EBGs, and demonstrations of filters, transitions, MMIC packaging, corporate distribution networks, and slot and horn array antennas. The technology demonstrators cover the three different versions of gap waveguides: groove gap waveguide, ridge gap waveguides and microstrip gap waveguides.
\end{abstract}

Index Terms - Gap waveguides, packaging, filters, millimeterwaves, submillimeterwaves, AMC, EBG, soft and hard surfaces, metamaterials.

\section{INTRODUCTION}

The concept of soft and hard surfaces was introduced in 1988 [1] and 1990 [2], based on the characteristics of the corrugated surfaces used in corrugated horn antennas. The soft surface is related to the more common EBG surface appearing in the literature since year 2000, but it is an anisotropic and wideband version of it, stopping waves along the surface in one direction only. The hard surface was used to realize cloaking in 1997, referred to as reduction of forward scattering [3] or blockage reduction (and even superstealth in a conference paper). This is ten years before cloaking became a popular research topic. The relations between soft and hard surfaces and EBG surfaces and in particular artificial magnetic conductors was described in 2005 [4]. Actually, the soft and hard surfaces are ideally grids of parallel perfect electric conducting (PEC) and perfect magnetic conducting strips, i.e. PEC/PMC strip grids.
The gap waveguides were invented in 2008. The basic design was inspired by works on hard surfaces at Polytechnic University of Valencia (UPV) [5], and they also contributed to the first paper defining the concept [6]. The principle idea is to generate a stopband between two parallel plates, so that no parallel-plate modes can propagate, and then to introduce grooves or conductive ridges or metal strips along which waves can propagate. The stopband characteristics in other directions make the waves confined to the groove, ridge or strip. This also then defines the three different gap waveguides that exist: groove, ridge and microstrip gap waveguides (Figure 1).

The parallel-plate stopband can be realized in many ways, by many types of periodic elements. The easiest way to explain it is to let one plate be a PMC and the other a PEC. Then, no parallel-plate modes can propagate as long as the gap between then plates is smaller than quarter wavelength. When introducing PEC strips in the PMC surface, we get the gap waveguide transmission line. The high surface impedance of artificial magnetic conductors (AMCs) has normally very narrow bandwidth. Still, a stopband for parallel-plate modes can be easily realized over a significant octave 2:1 bandwidth. Some of the periodic elements used to design the stopband so far are studied in [7], but others have also been published. The surface with most potential in $\mathrm{mm}$ and submm-wave region is made of periodic metal pins/posts(nails.

The rest of this paper overviews what we have developed so far.

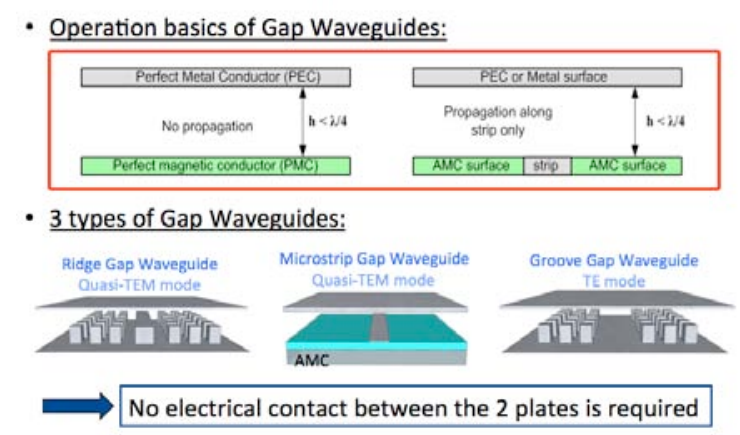

Figure 1. Illustration of the principle of operation of the gap waveguides (upper), and three different types of gap waveguides (lower). The AMC in the middle microstrip gap waveguide case can also be realized with pins like in the two other cases. 


\section{DEMONSTRATIONS OF LOW LOSS UP TO $260 \mathrm{GHZ}$}

The topologies of ridge and microstrip gap waveguide circuits are similar to that of microstrip circuits, but the losses are much lower. One reason is of course that there is no dielectric in the gap waveguide cases, but this does normally not make up more than $25 \%$ of the total loss as seen in Figure 2 . The low loss is coming from the increased dimensions. Microstrip lines must be used on thin substrates in order to avoid surface waves and radiation, whereas in ridge and microstrip gap waveguides the airgap can be two and even four times larger without any similar problems, and this reduces the conductive losses from $4.5 \mathrm{~dB} / \mathrm{dm}$ to $1 \mathrm{~dB} / \mathrm{dm}$, as seen in Figure 2, and there is no dielectric losses.

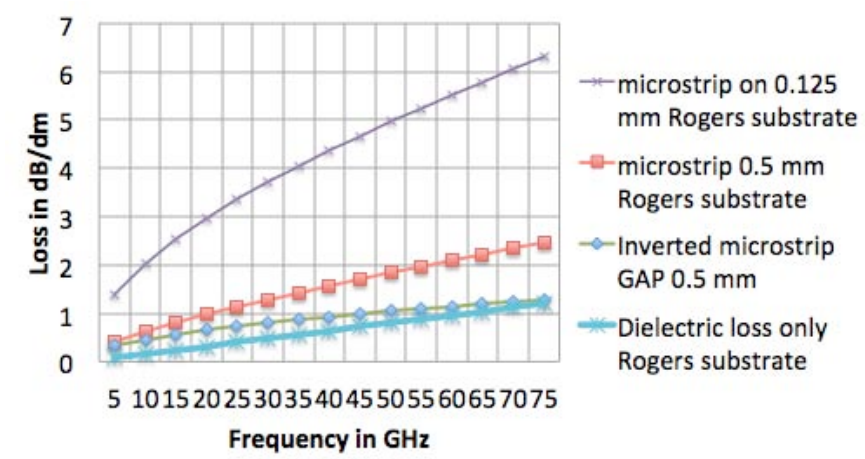

Figure 2. Graph showing the total losses in microstrip line on thin $0.125 \mathrm{~mm}$ lowloss substrate, thick $0.5 \mathrm{~mm}$ lowloss substrate, and for comparison the contribution only from dielectric losses, which is independent of substrate thickness and line widths. With ridge and microstrip gap waveguides we can use air gaps that are much larger than the substrate thickness without problems with surface waves or radiation, and thereby we can get losses of $1.0 \mathrm{~dB} / \mathrm{dm}$ compared to $5.5 \mathrm{~dB} / \mathrm{dm}$ for a microstrip circuit at $60 \mathrm{GHz}$.
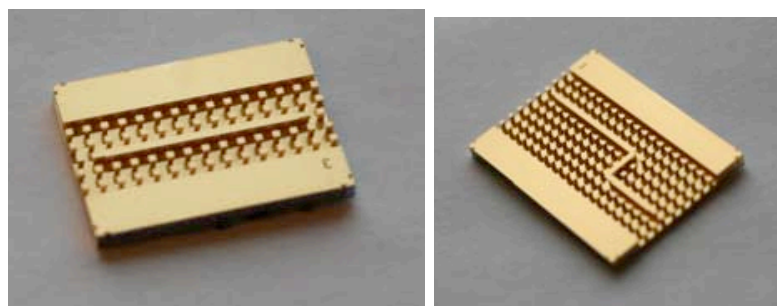

Figure 3. Gap waveguide resonator for measuring losses, and a demonstrator with two $90 \mathrm{deg}$ bends, both realized for $100 \mathrm{GHz}$ by micromachining on Gold-sputtered Silicon. Similar circuits exist at

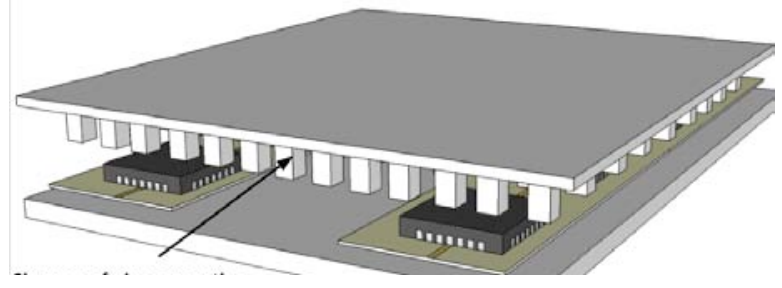

$260 \mathrm{GHz}$. The measured results confirm the theory.

Figure 4. Tx and Rx MMIC amplifier chains packaged by lid of nails. The configuration has also been built and measured.
The topology of groove gap waveguide components is similar to that of rectangular waveguide components. We have also shown that the losses of the groove gap waveguides are about $20 \%$ larger than of normal rectangular waveguides.

The experimental studies of the losses are documented in [8] for microwave frequencies, and in [9] for frequencies above $100 \mathrm{GHz}$. The latter higher-frequency gap waveguides have been realized by micromaching.

\section{PACKAGING}

\section{A. AMC gap waveguide packaging}

The packaging by an AMC pin lid was first demonstrated in [10], and it has later been used successfully also to package microstrip filters [11]. At frequencies below $10 \mathrm{GHz}$ the pins may with advantage be replaced by springs [12] or zigzag wires [13].

\section{B. Numerical PMC prepackaging to reduce computation time}

The pins, springs or zigzag wires can initially in a design process be replaced by a PMC lid to reduce computational volume and time. This results in a complete new procedure of designing high-frequency circuits and modules. They can be initially designed with a PMC lid, thereby removing cavity resonances that otherwise would appear with with PEC or metal lid, and the PMC lid can later be replaced by a realized lid consisting of pins or other periodic elements. This numerical approach is referred to as PMC prepackaging [14] and reduce the computational effort of the initial design, but the design has to be fine-tuned after introduction of the realization of the PMC.

\section{Packaging of MMICs}

A major development during 2012 was the packaging of Tx and Rx amplifier chains [15], see Figure 4. It was found that the lid of nails packaging makes it possible to use the amplifiers for $25 \mathrm{~dB}$ larger output power than with normal packaging using metal cavities with absorbers in the roof. Also, a minimum isolation between the Tx and $\mathrm{Rx}$ amplifier chains of $78 \mathrm{~dB}$ was achieved without any solid metal wall between the Tx and Rx sides. The $78 \mathrm{~dB}$ isolation is entirely due to the parallel-plate cut-off created by the pins.

\section{FILTERS AND ANTENNAS}

The gap waveguides have low losses, so they naturally can be applied to make high-Q filters. This was first demonstrated in [16], and thereafter a $38 \mathrm{GHz}$ filter was made according to commercial radio link specifications and successfully tested, see Figure 5. The manufacturing cost is not yet comparable with rectangular waveguide filters, but it has shown better temperature stability, and manufacturing should become 
cheaper when a good way to realize the pins have been developed [17].

We have now started to realize gap waveguide antennas. The first linear ridge gap waveguide slot arrays with a corporate feeding is presented in [18], and a planar $2 \times 2$ horn array in microstrip gap waveguide is reported in [19]. Some larger $4 \times 4$ example of the horn antenna array is shown in Figure 5a, and in addition a $2 \times 2$ ridge gap waveguide slot array with $25 \%$ bandwidth in Figure $5 \mathrm{~b}$. The simulations and measurements will soon be reported.

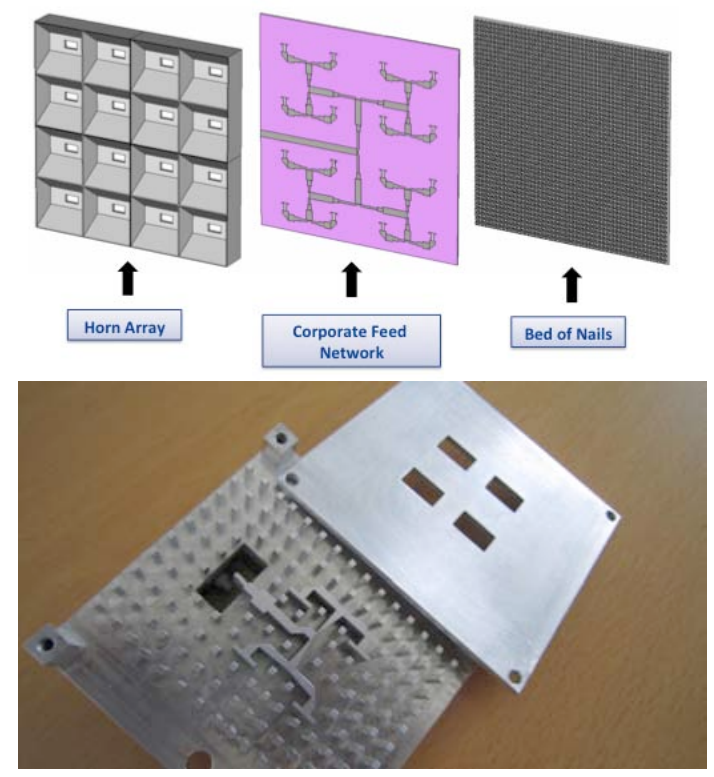

Figure 5. Examples of gap waveguide antennas. $4 \times 4$ microstrip gap waveguide slot array (upper), and $2 \times 2$ ridge gap waveguide array (lower).

\section{CONCLUSION AND ACKNOWLEDGEMENT}

The research on gap waveguides has since it was started in 2008 progressed well, and the technology has many attractive features that have major advantages above $30 \mathrm{GHz}$.

The part of this research done at Chalmers has been supported by Swedish Foundation for Strategic Research (SSF), Swedish Governmental Agency for Innovation Systems (VINNOVA) and Swedish Research Council VR.

\section{REFERENCES}

[1] P. S. Kildal, "Definition of artificially soft and hard surfaces for electromagnetic waves," Electronics Letters, vol. 24, pp. 16870, 1988 .

[2] P. S. Kildal, "Artificially soft and hard surfaces in electromagnetics," IEEE T-AP, vol. 38, pp. 1537-44, 1990.

[3] P. S. Kildal, A. A. Kishk, and A. Tengs, "Reduction of forward scattering from cylindrical objects using hard surfaces," IEEE T-AP, vol. 44, pp. 1509-20, 1996.
[4] P.-S. Kildal, A. A. Kishk, and S. Maci, "Special issue on artificial magnetic conductors, soft/hard surfaces, and other complex surfaces," IEEE T-AP, vol. 53, pp. 2-7, jan. 2005.

[5] E. Alfonso, A. Valero-Nogueira, J. I. Herranz, and F. Vico, "Optimization of hard surfaces within parallel plate waveguides applied to slot-array antennas," in EuCAP 2007, 11-16 Nov. 2007, Stevenage, UK, 2007, p. 6 pp.

[6] P. S. Kildal, E. Alfonso, A. Valero-Nogueira, and E. RajoIglesias, "Local metamaterial-based waveguides in gaps between parallel metal plates," IEEE Antennas and Wireless Propagation Letters, vol. 8, pp. 84-7, 2009.

[7] E. Rajo-Iglesias and P. S. Kildal, "Numerical studies of bandwidth of parallel-plate cut-off realised by a bed of nails, corrugations and mushroom-type electromagnetic bandgap for use in gap waveguides," IET Microwaves, Antennas and Propagation, vol. 5, pp. 282-9, 2011.

[8] E. Pucci, A. U. Zaman, E. Rajo-Iglesias, P.-S. Kildal, and A. Kishk, "Study of Q-Factors of Ridge and Groove Gap Waveguide Resonators," IET Microwaves, Antennas and Propagation, accepted for publication in April 2013.

[9] S. Rahiminejad, A. U. Zaman, E. Pucci, H. Raza, V. Vassilev, S. Haasl, P. Lundgren, P.-S. Kildal, and P. Enoksson, "Micromachined Ridge Gap Waveguide and Resonator forMillimeter-Wave Applications," Sensors and Actuators A: Physical, vol. 186, October 2012.

[10] E. Rajo-Iglesias, A. U. Zaman, and P. S. Kildal, "Parallel plate cavity mode suppression in microstrip circuit packages using a lid of nails," IEEE Microwave and Wireless Components Letters, vol. 20, pp. 31-3, 2010.

[11] A. Algaba Brazález, A. U. Zaman, and P.-S. Kildal, "Improved Microstrip Filters Using PMC Packaging by Lid of Nails," IEEE T-CPMT, vol. 2, July 2012.

[12] E. Rajo-Iglesias, P.-S. Kildal, A. U. Zaman, and A. Kishk, "Bed of Springs for packaging of microstrip circuits in the microwave frequency range," IEEE T-CPM, vol. 2, October 2012.

[13] E. Rajo-Iglesias, E. Pucci, A. Kishk, and P.-S. Kildal, "Suppression of parallel plate modes for low frequency microstrip circuit packaging using lid of printed zigzag wires," IEEE MWCL, accepted for publication in, April 2013.

[14] A. Kishk, A. U. Zaman, and P.-S. Kildal, "Numerical Prepackaging with PMC lid - Efficient and Simple Design Procedure for Microstrip Circuits including the Packaging," ACES journal, vol. 2, May 2012.

[15] A. U. Zaman, T. Vukusic, M. Alexanderson, and P. S. Kildal, "Gap Waveguide PMC Packaging for Improved Isolation of Circuit Components and MMICs in High Frequency Microwave Modules," IEEE T-CPMT, accepted for publication in April, 2013.

[16] A. U. Zaman, A. Kishk, and P.-S. Kildal, "Narrow-band microwave filter using high $\mathrm{Q}$ groove gap waveguide resonators with manufacturing flexibility and no sidewalls," IEEE T-CPMT, vol. 2, pp. 1882-1889, November 20122012.

[17] E. Alfonso Alos, A. U. Zaman, and P.-S. Kildal, "Ka-band Gap Waveguide Coupled-Resonator Filter for Radio Link Diplexer Application," IEEE T-CPMT, Accepted for publication in, November 2012.

[18] A. U. Zaman and P.-S. Kildal, "Linear Slot Array Design in Ridge Gapwaveguide Technology," EuCAP 2013, Gothenburg, 8-12 April, 2013.

[19] E. Pucci, E. Rajo-Iglesias, J.-L. Vazquez-Roy, and P.-S. Kildal, "Design of a four-element horn antenna array fed by inverted microstrip gap waveguide," in IEEE International Symposium on Antennas and Propagation, Orlando, USA, 2013. 